Short communication

\title{
Tachycardiomyopathy: A case report and review of literature
}

\author{
Anunay Gupta, K.K. Talwar * \\ Department of Cardiology, Max Heart and Vascular Institute, Max Saket, New Delhi, India
}

\section{A R T I C L E I N F O}

\section{Article history:}

Received 23 August 2017

Received in revised form 27 September 2017

Accepted 29 September 2017

Available online 5 October 2017

\section{Keywords:}

tachycardiomyopathy

PJRT

\begin{abstract}
A B S T R A C T
Tachycardiomyopathy is well-recognized reversible form of myocardial dysfunction caused by chronic arrhythmias. Treatment of arrhythmia leads to recovery of myocardial function. Hence, it is of utmost importance to look for and identify arrhythmias in every patient presenting with myocardial dysfunction without any apparent etiology. We hereby present a case of persistent junctional reciprocating tachycardia with cardiomyopathy whose left ventricular function reverted to normal in three months following successful radiofrequency ablation of tachycardia.

(c) 2017 The Society of Cardiovascular Academy. Production and hosting by Elsevier B.V. All rights reserved. This is an open access article under the CC BY-NC-ND license (http://creativecommons.org/licenses/by-nc-nd/4.0/).
\end{abstract}

\section{Introduction}

Tachycardiomyopathy is an uncommon, however wellrecognized entity caused by long standing tachycardia which is usually treatable, reversible and hence have excellent prognosis. ${ }^{1}$ Tachycardiomyopathy is reported and seen with all type of tachyarrhythmias. Supraventricular arrhythmias such as atrial fibrillation, atrial flutter, atrial tachycardias and reentrant supraventricular tachycardia (SVT) can lead to tachycardiomyopathy. Ventricular arrhythmias rarely lead to tachycardiomyopathy since this arrhythmia is usually associated with some form of underlying structural heart disease. We hereby report a case of tachycardiomyopathy due to paroxysmal junctional reciprocating tachycardia (PJRT) which was initially missed due to its paroxysmal nature and was later on cured with successful ablation of tachycardia.

\section{Case report}

A fourteen year old girl presented first in 1999 with history of shortness of breath. There was a history of fever 2 months back along with easy fatiguability. She had heart rate of $110 / \mathrm{min}$ with blood pressure of 100/60 mm Hg. Electrocardiogram was interpreted as sinus tachycardia. Echocardiogram revealed a severely depressed left ventricular ejection fraction of approximately $25-30 \%$ with global hypokinesia.

\footnotetext{
* Corresponding author at: Department of Cardiology, Max Heart and Vascular Institute, Saket, New Delhi, India.

E-mail address: kktalwar@hotmail.com (K.K. Talwar).

Peer review under responsibility of The Society of Cardiovascular Academy.
}

She was given a diagnosis of dilated cardiomyopathy probably postviral myocarditis and underwent endomyocardial biopsy which showed non-specific hypertrophy, nuclear enlargement without any evidence of myocarditis. She had recurrent palpitations. Hence keeping a possibility of tachycardiomyopathy patient underwent electrophysiology study (EPS). During EPS, tachycardia could not be induced this time. Patient was started on oral amiodarone, beta blocker and digitalis along with decongestive therapy and followed. Her LV function improved (LVEF - 55\%) over next few months and subsequently she was lost to follow up.

Subsequently the patient presented again in 2006 with worsening dyspnea, palpitations, fever and cough. Echocardiography showed LVEF of $20-25 \%$. Her heart rate was high $160 / \mathrm{min}$. It was noted, however, that she had evidence of an incessant narrow complex supraventricular tachycardia (SVT) on her electrocardiogram (ECG). There was a long RP narrow complex SVT with inverted P waves in leads II, III, and aVF with upright $\mathrm{p}$ in V1 (Fig. 1). She was treated with antibiotics and after 10 days underwent electrophysiology study. During the study patient was having incessant tachycardia. It was a narrow complex tachycardia with heart rate of $160 / \mathrm{min}$. It was similar to the baseline ECG. It was a long RP tachycardia with earliest retrograde A at CS 9-10 with a long local VA interval of $170 \mathrm{~ms}$ (Fig. 2). Tachycardia was entrained by ventricular pacing at right ventricle apex which showed $\mathrm{V}-\mathrm{A}-\mathrm{V}$ sequence at termination of $\mathrm{V}$-pacing ruling out atrial tachycardia. Postpacing interval minus tachycardia cycle length of $44 \mathrm{~ms}$ was suggestive of pathway. His refractory ventricular premature complex (VPC) resetted the tachycardia. Earliest A was mapped at inferior rim of CS os. On basis of these findings diagnosis of orthodromic reciprocating tachycardia utilizing a slowly conducting posteroseptal accessory pathway i.e. PJRT was made. Tachycardia terminated within $5 \mathrm{~s}$ of 


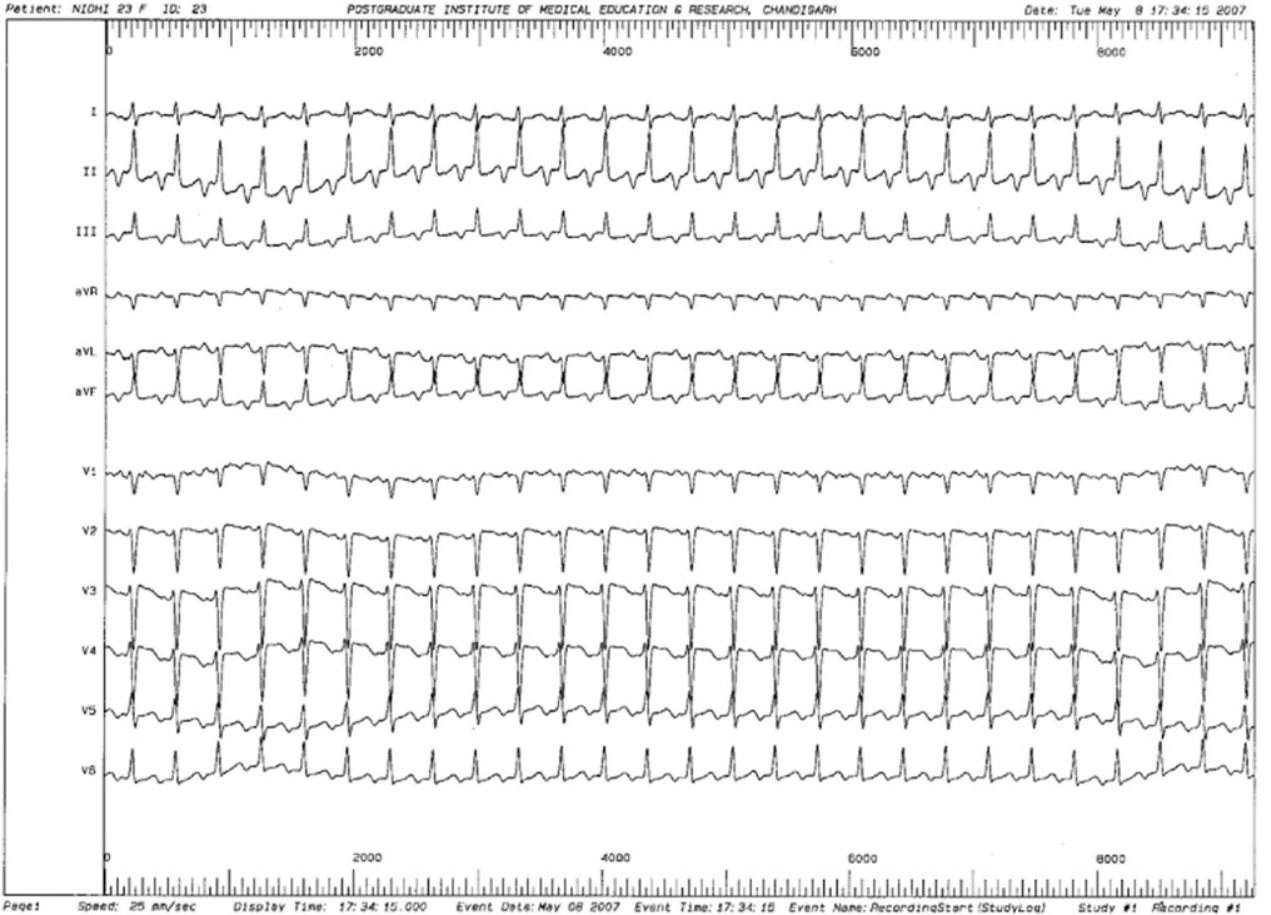

Fig. 1. Twelve lead electrocardiogram in electrophysiology lab showing narrow complex tachycardia with HR 160/min with inverted P in II, III, AVF and upright P in V1.

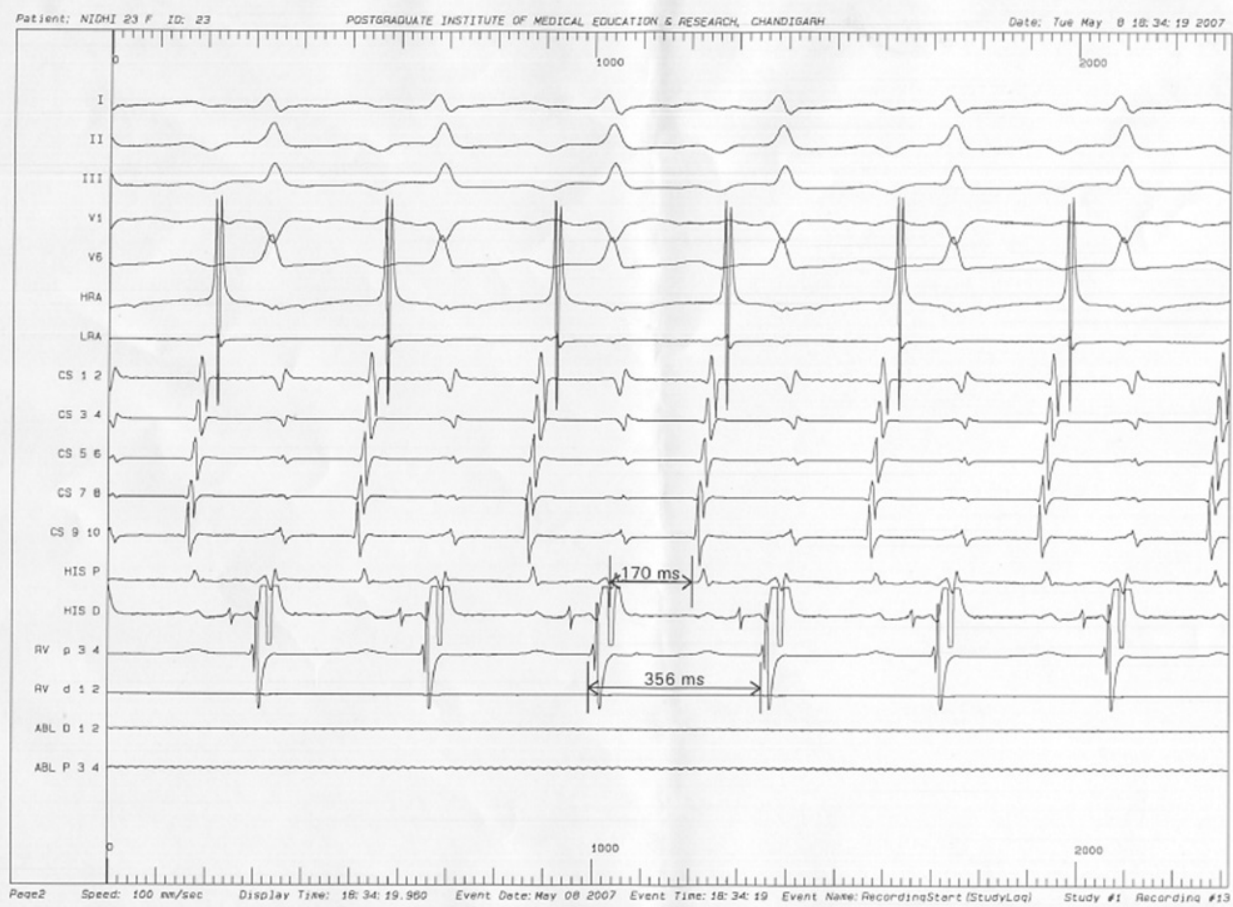

Fig. 2. Tracing showing long RP tachycardia with earliest retrograde A at CS 9-10 with long local VA interval of $170 \mathrm{~ms}$ 


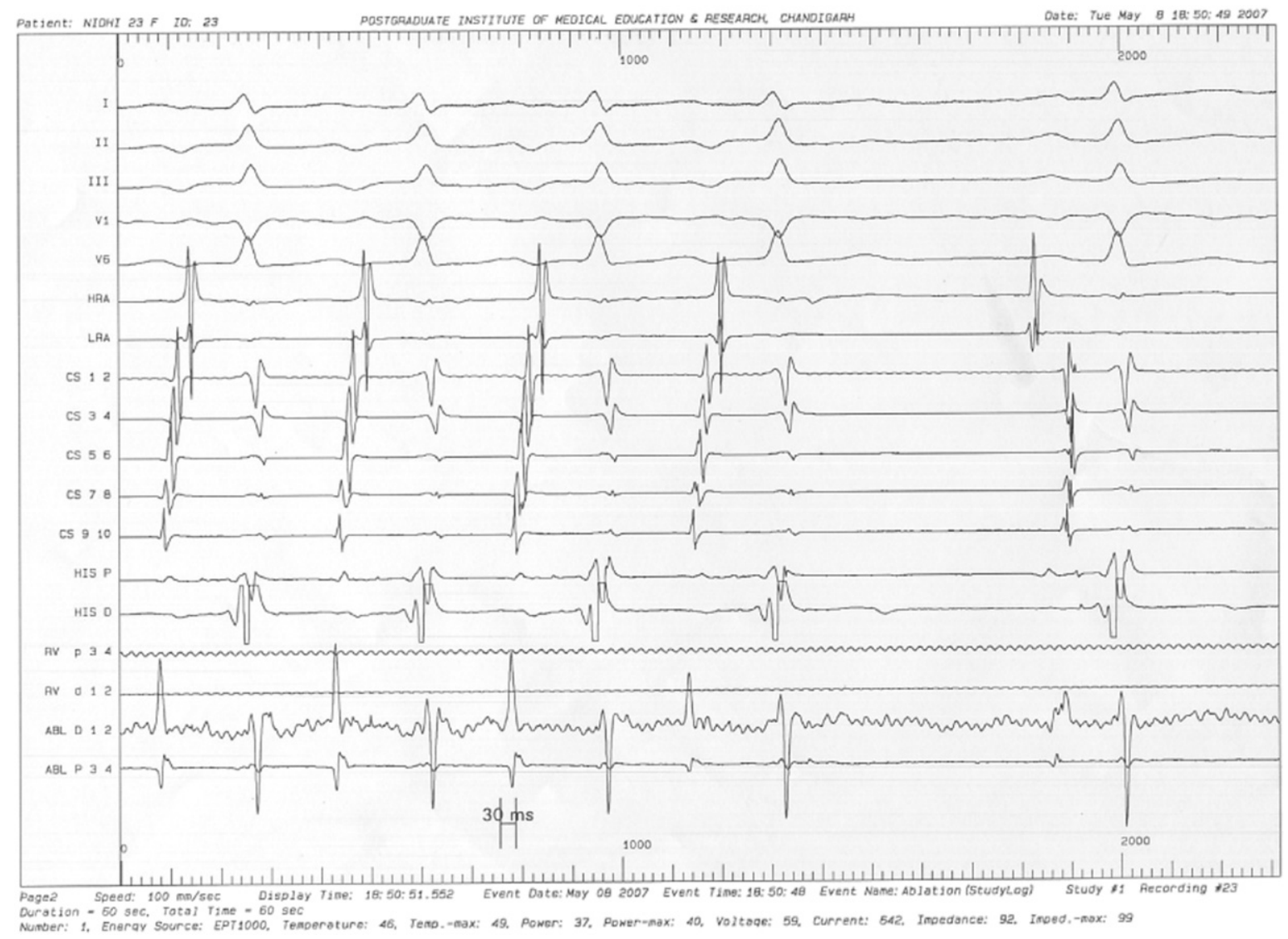

Fig. 3. Earliest A was mapped at inferior rim of CS os. Termination of tachycardia occurred within $5 \mathrm{~s}$ of radiofrequency burn.

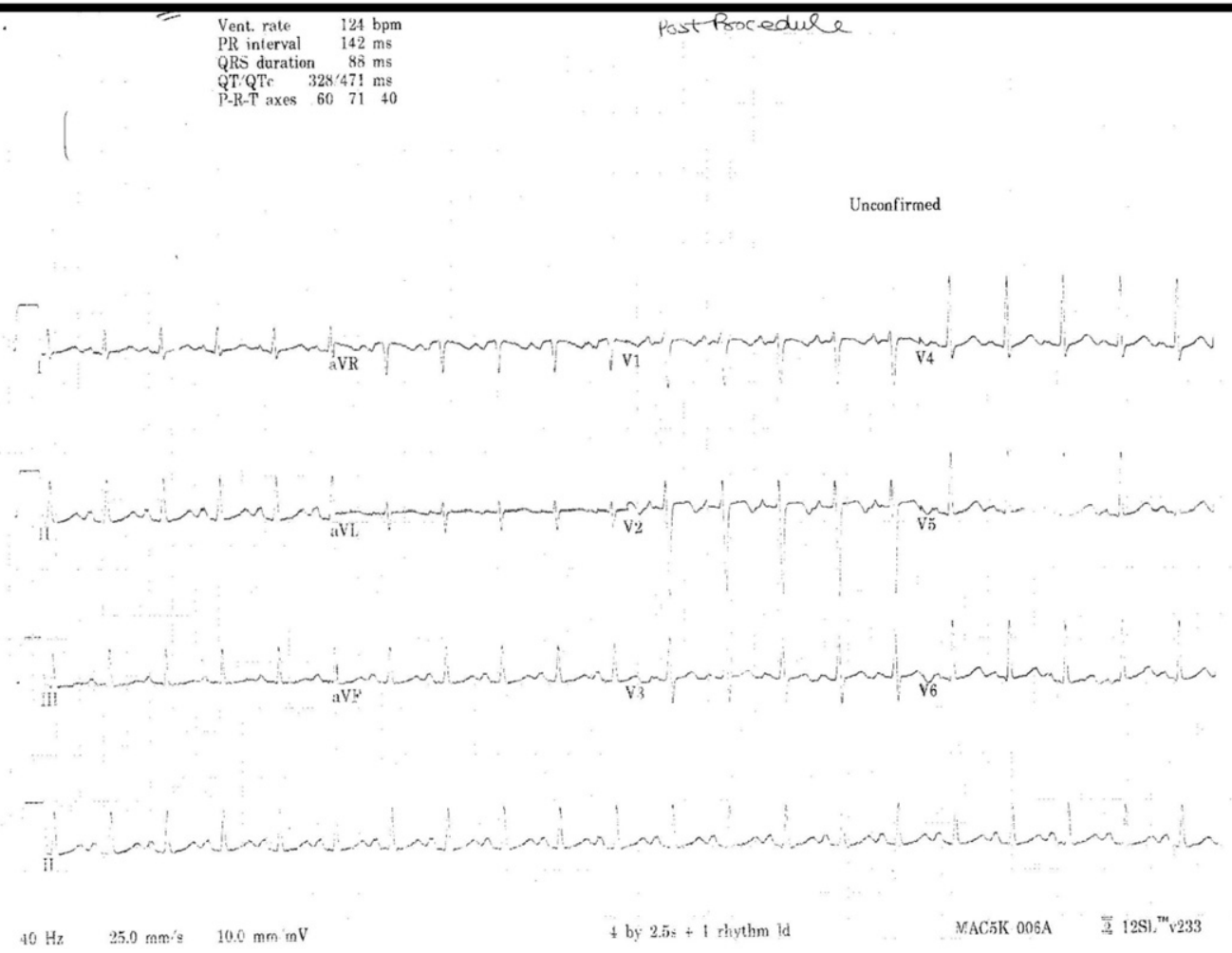

Fig. 4. Post-ablation twelve lead electrocardiogram which shows normal sinus rhythm. 
radiofrequency ablation (temperature of $60^{\prime} \mathrm{C}$, power of $45 \mathrm{~W}$ ) at site of earliest retro A (Fig. 3). The lesion was continued for $40 \mathrm{~s}$.

Patient was discharged four days later in normal sinus rhythm (Fig. 4). She came to follow up after three months. Her Left ventricular function has improved to $50 \%$ and she was NYHA Class I.

\section{Discussion}

PJRT and atrial tachycardia accounts for the most of the causes of tachycardiomyopathy in children. Atrial fibrillation and atrial flutter also leads to tachycardiomyopathy if ventricular rates are not controlled. Ventricular arrhythmias which cause tachycardiomyopathy are usually idiopathic in nature. Sustained monomorphic VT rarely causes tachycardiomyopathy as it is more often associated with pre-existing structural heart disease. Tachycardiomyopathy is seen with very frequent premature ventricular contractions, ventricular tachycardia of outflow tract of right ventricle and fascicular tachycardia., ${ }^{2,3} \mathrm{PJRT}$ is usually characterized by incessant form of supraventricular tachycardia and leads to tachycardiomyopathy. However it can be paroxysmal and can be missed as it happened in our patient. ${ }^{4}$ PJRT is a narrow QRS tachycardia with long RP interval which is also seen in atrial tachycardia and atypical AVNRT, hence requires electrophysiological study for precise diagnosis. It is more common in children than in adults. Recognition of PJRT as a cause of non-ischemic cardiomyopathy is important as its ablation leads to dramatic improvement in left ventricular function. Medical therapy is usually ineffective in controlling heart rate. Posteroseptal accessory pathways commonly seen in PJRT as seen in our case these are situated near the os of coronary sinus are high risk area of ablation due to proximity to left circumflex atery. ${ }^{5}$ Cryoablation is a suitable alternative to radiofrequency ablation with minimal risk to damage to coronary artery. ${ }^{6}$

There is usually a rapid clinical improvement after successful ablation of tachycardia. By three months there is usually recovery of left ventricular function as also seen in our patient. ${ }^{7}$ In a recent study majority of echocardiographic parameters including ejection fraction improved significantly after treatment. However, left ventricular dimensions and volumes remained significantly elevated when compared with control subjects, indicating persistence of negative LV remodeling, even after appropriate treatment and normalization of EF at a mean follow-up of 14 months. ${ }^{8}$ Hence patients should continue to be monitored and treated with anti-reverse remodeling drugs like beta-blockers and ACE inhibitors.

Hence, we have described a case of PJRT related tachycardiomyopathy which was missed initially due to its paroxysmal nature. Diagnosis of Tachycardiomyopathy should be considered in the presence of persistent supraventricular or ventricular tachyarrhythmia with heart failure.

\section{References}

1. Packer DL, Bardy GH, Worley SJ, et al. Tachycardia-induced cardiomyopathy: a reversible form of LV dysfunction. Am J Cardiol 1986;57(8):563-570.

2. Vijgen J, Hill P, Biblo LA, Carlson MD. Tachycardia-induced cardiomyopathy secondary to right ventricular outflow tract ventricular tachycardia: improvement of left ventricular systolic function after radiofrequency catheter ablation of the arrhythmia. $J$ Cardiovasc Electrophysiol 1997;8(4):445-450.

3. Singh B, Kaul U, Talwar KK, Wazir HS. Reversibility of "tachycardia induced cardiomyopathy" following the cure of idiopathic left ventricular tachycardia using radiofrequency energy. Pacing Clin Electrophysiol 1996;19(9):1391-1392.

4. Noda T, Shimizu W, Suyama K, et al Coexistence of the permanent form of junctional reciprocating tachycardia and atrial tachycardia. Circ J 2005;69(8):1003-1006.

5. Schneider HE, Kriebel T, Gravenhorst VD, Paul T. Incidence of coronary artery injury immediately after catheter ablation for supraventricular tachycardias in infants and children. Heart Rhythm 2009;6(4):461-467.

6. Aoyama H, Nakagawa H, Pitha JV, et al. Comparison of cryothermia and radiofrequency current in safety and efficacy of catheter ablation within the canine coronary sinus close to the left circumflex coronary artery. J Cardiovasc Electrophysiol 2005;16(11): 1218-1226.

7. Jeong Y-H, Choi K-J, Song J-M, et al. Diagnostic approach and treatment strategy in tachycardia-induced cardiomyopathy. Clin Cardiol 2008;31(4):172-178.

8. Dandamudi G, Rampurwala AY, Mahenthiran J, Miller JM, Das MK. Persistent left ventricular dilatation in tachycardia-induced cardiomyopathy patients after appropriate treatment and normalization of ejection fraction. Heart Rhythm 2008;5(8):1111-1114. 